\title{
Contribution of catechol- $O$-methyltransferase Val158Met polymorphism to endometrial cancer risk in postmenopausal women: a meta-analysis
}

\author{
G. Lin ${ }^{1 *}$, J. Zhao ${ }^{2 *}$, J. Wu ${ }^{2}$, R. Andreevich $\mathrm{O}^{1}$, W.-H. Zhang ${ }^{2 *}$, Y. Zhang ${ }^{2,3}$ \\ and $\mathrm{L}$. Yu ${ }^{1}$ \\ ${ }^{1}$ The State Key Laboratory of Genetic Engineering, Institute of Genetics, \\ Fudan University, Shanghai, China \\ ${ }^{2}$ Department of Infectious Diseases, Huashan Hospital, \\ Fudan University, Shanghai, China \\ ${ }^{3}$ Department of Molecular Microbiology and Immunology, \\ Bloomberg School of Public Health, John Hopkins University, \\ Baltimore, MD, USA \\ *These authors contributed equally to this study. \\ Corresponding authors: Y. Zhang / L.Yu \\ E-mail: pharmlife2002@yahoo.com / longyu@fudan.edu.cn
}

Genet. Mol. Res. 12 (4): 6442-6453 (2013)

Received November 5, 2012

Accepted April 18, 2013

Published December 10, 2013

DOI http://dx.doi.org/10.4238/2013.December.10.5

\begin{abstract}
Catechol- $O$-methyltransferase (COMT) is a critical enzyme to detoxify the carcinogenic catechol estrogen and the Val158Met polymorphism of COMT could influence its enzymatic activity. Recent epidemiological studies have investigated the correlation of COMT Val158Met polymorphism with endometrial cancer risk; however, the results are inconsistent. To better evaluate the role of COMT Val158Met in endometrial carcinogenesis, we performed this meta-analysis, considering menopausal status, study quality, ethnicity, and source of controls. Eight eligible studies
\end{abstract}


including 5109 subjects were collected from PubMed, CNKI, and Chinese Biomedicine Database (updated until September 21, 2012). Although no obvious associations were detected between COMT Val158Met and endometrial cancer susceptibility in the pooled analysis, we noted significantly decreased endometrial cancer risk for Val/Met versus Val/Val, and Met/Met + Val/Met versus Val/Val genetic models in the postmenopausal female $(\mathrm{OR}=0.795,95 \% \mathrm{CI}$ $=0.656-0.962, \mathrm{P}=0.019 ;$ and $\mathrm{OR}=0.819,95 \% \mathrm{CI}=0.683-0.983$, $\mathrm{P}=0.032$; respectively), and similar results existed in high-quality studies $(\mathrm{OR}=0.835,95 \% \mathrm{CI}=0.726-0.961, \mathrm{P}=0.012$; and $\mathrm{OR}=$ $0.853,95 \% \mathrm{CI}=0.747-0.974, \mathrm{P}=0.019$; respectively). However, no evidence of association was noted in different ethnic groups and sources of controls. In conclusion, our results suggested that the COMT Val/Val genotype might act as a potential endometrial cancer risk factor in postmenopausal women. Further studies are needed to investigate the interactions between COMT Val158Met polymorphism and endometrial cancer in a specific population.

Key words: COMT; Polymorphism; Endometrial cancer; Meta-analysis

\section{INTRODUCTION}

Endometrial cancer is a gynecological malignancy prevalent worldwide and the death rate attributed to it has increased in recent years (Sorosky, 2008). Various risk factors are involved in the development of endometrial cancer, such as prior exogenous estrogen administration, obesity, nulliparity, diabetes, genetic factors, etc. (Sorosky, 2008). Estrogen biosynthesis and metabolism are closely associated with tumorigenesis, and the functional gene polymorphisms regulating these processes can influence the levels of estrogen and intermediate products, which may potentially contribute to the differences in individual susceptibility to endometrial cancer (Huber et al., 2002).

Three major estrogens exist in vivo: estrone (E1), estradiol (E2), and estriol (E3). These estrogens can be hydroxylated into 2-hydroxy- and 4-hydroxy-estrone/estradiol (2/4-OH catechol estrogens) (Zhu and Conney, 1998a), and then, the 2/4-OH catechol estrogens are inactivated by methylation to form 2/4-methoxy-estrone/estradiol (2/4-MeOE1/E2). The methylation modification is catalyzed via catechol-O-methyltransferase (COMT) (Ball et al., 1972). If the methylation reaction is incomplete, these catechol estrogens will be oxidized to semiquinones and quinines; this process can produce reactive oxygen species, causing DNA damage and tumor initiation (Cavalieri et al., 1997). Therefore, COMT plays the critical role of detoxifying carcinogenic catechol estrogens. Moreover, the 2-methoxy-estradiol (2-MeO-E2) metabolite catalyzed by COMT can inhibit the proliferation and migration of endothelial cells, angiogenesis and cytotoxin, and can induce apoptosis to suppress carcinogenesis (Zhu and Conney, 1998b). A functional polymorphism exists in COMT gene, the $\mathrm{G} \rightarrow \mathrm{A}$ transition at codon158 (rs4680) in exon 4 , leading to the substitution of valine to methionine (known as Val158Met). The homozygous mutant Met/Met genotype has been reported to decrease the COMT methylation 
activity by $3 \sim 4$ folds compared with wild-type Val/Val genotype; the heterozygous Val/ Met genotype has intermediate activity (Dawling et al., 2001). Since COMT Val158Met polymorphism can influence the enzymatic activity and may further alter the accumulation of circulating levels of carcinogenic catechol estrogens, therefore, Val158Met has long been the focus of hormone-related cancers such as prostate (Suzuki et al., 2007), breast (He et al., 2012), and endometrial cancer.

In recent years, many studies have investigated the relationship between COMT Val158Met polymorphism and endometrial cancer susceptibility (McGrath et al., 2004; Zimarina et al., 2004; Doherty et al., 2005; Tao et al., 2006; Liu et al., 2007; Szyllo et al., 2007; Zhao et al., 2007; Hirata et al., 2008a,b; Li et al., 2010); however, the results are controversial. Zhao et al. (2007) found that COMT Val/Val carriers may have decreased endometrial cancer risk versus individuals with Met/Met genotype, whereas Doherty et al. (2005) demonstrated that COMT Val/Met and Met/Met carriers may show only a slight reduction in the risk. In contrast, others have not found any correlation (McGrath et al., 2004; Zimarina et al., 2004; Tao et al., 2006; Liu et al., 2007; Szyllo et al., 2007; Hirata et al., 2008a,b; Li et al., 2010). The inconsistent conclusions may be due to a possible minor effect of the polymorphism on endometrial cancer or the small sample size in single studies with inadequate statistical power of complex traits. Meta-analysis is a powerful statistical tool pooling different studies to overcome deficiencies such as small sample size, which will provide more reliable results. So far, no meta-analysis has assessed the association between COMT Val158Met polymorphism and endometrial cancer risk. Here, we performed a metaanalysis of eight eligible studies, including 2296 cases and 2813 controls, to evaluate the correlation more precisely.

\section{MATERIAL AND METHODS}

\section{Publication search}

To identify all studies that investigated the association between COMT Val158Met polymorphism and endometrial cancer risk, we conducted a literature search in PubMed, CNKI (Chinese National Knowledge Infrastructure), and Chinese Bio-medicine Database by using following terms: "catechol-O-methyltransferase", "COMT", "polymorphism", "endometrial cancer", as well as their combinations. References cited in the retrieved and review articles were also searched manually. There were no language restrictions to the publications and the last update was dated September 21, 2012.

\section{Inclusion and exclusion criteria}

Eligible studies were selected on the basis of the following criteria: (a) full-text articles about COMT Val158Met polymorphism and endometrial cancer risk; (b) case-control studies with available data for estimating the odds ratio (OR) with $95 \%$ confidence interval (CI); and (c) if the studies used overlapping case-control data, only the recent and most complete studies were included in our meta-analysis. The main exclusion criteria were (a) not case-control studies, (b) control population including patients with tumors, and (c) duplication of a previous publication. 


\section{Data extraction}

Two investigators (G. Lin and J. Zhao) independently extracted information from all eligible publications to reduce bias in data collection. The data comprised the first author's surname, year published, country of origin, ethnicity, sample source, specimens of cases, sample size, and methods for detecting COMT Val158Met genotypes, total number of cases and controls, numbers of cases and controls with $\mathrm{Val} / \mathrm{Val}, \mathrm{Val} / \mathrm{Met}$, and Met/Met genotypes, respectively.

\section{Quality score assessment}

The quality of these studies was also evaluated independently by the same two investigators according to the predefined quality assessment rules in Table 1 . The scores were determined using both traditional epidemiological consideration and cancer genetic issues (Jiang et al., 2010). Checking and discussion between the two investigators led to consensus on all the items. The total score ranged from 0 (worst) to 15 (best). Papers scoring $<10$ were classified as "low quality" and those scoring $\geq 10$ as "high quality."

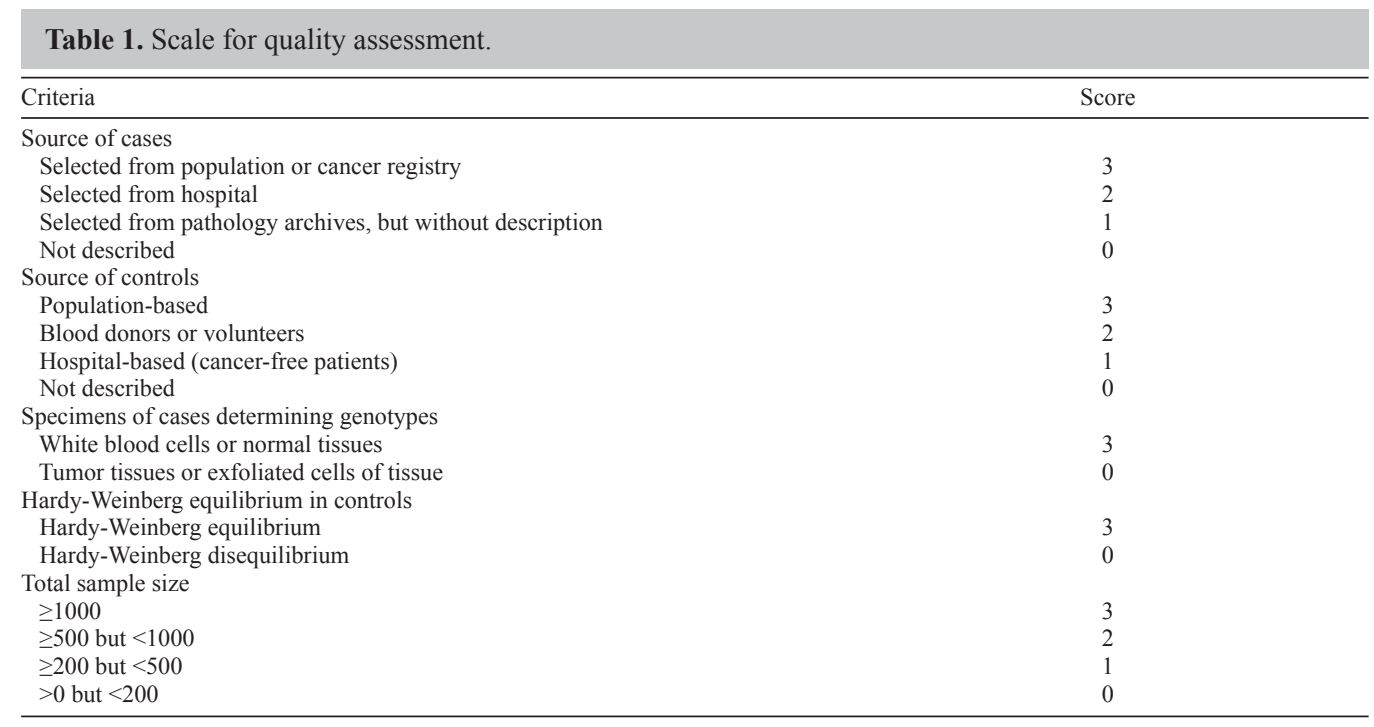

\section{Statistical analysis}

For each study, Hardy-Weinberg equilibrium (HWE) was analyzed with the Chi square-based test. The strength of the association between COMT Val158Met polymorphism and endometrial cancer risk was assessed by $\mathrm{OR}$ corresponding to $95 \% \mathrm{CI}$ according to the method described by Woolf (1955). Heterogeneity among studies was detected by Cochran's Chi square-based Q test (Higgins et al., 2003). If the result of heterogeneity test had a P value of $<0.1$, the random effect model using the DerSimonian and Laird method was employed to pool the results, which yielded wider CIs (DerSimonian and Laird, 1986). Otherwise, the fixed-effect model using the Mantel-Haenszel method was performed (Mantel and Haenszel, 
1959).

The significance of the pooled ORs was determined via the Z-test $(\mathrm{P}<0.05$ suggests significant association). Subgroup analysis was stratified by menopausal states (premenopausal and postmenopausal), study quality ("high" and "low"), ethnicity (Caucasian, Asian, and Mixed), and control sources (population-based and hospital-based). One-way sensitivity analyses were used to evaluate the stability of the results obtained. Publication bias was tested by both the Begg funnel plot (Begg and Mazumdar, 1994) and the Egger's linear regression test (Egger et al., 1997). If the funnel plot was asymmetric and the Egger test gave a $P$ value of $<0.05$, publication bias was assumed to exist (Egger et al., 1997). Then, the Duval and Tweedie non-parametric "trim and fill" methods were performed to adjust for it. All statistical tests were carried out with the Stata software version 10.0 (Stata Corporation, College Station, TX, USA) in this study.

\section{RESULTS}

\section{Study characteristics}

We identified 205 papers investigating the relationship between COMT polymorphisms and cancers from PubMed database (190) and from Chinese publications (15) as shown in Figure 1. After reviewing the titles and abstracts, 60 papers discussing COMT Val158Met polymorphism were selected, of which 14 studied endometrial cancer. When extracting data from the 14 candidate papers, five papers were excluded because four articles lacked case-control data and the population in one paper (Hirata et al., 2008b) overlapped with another included study (Hirata et al., 2008a). Thus, nine case-control studies met our inclusion criteria (McGrath et al., 2004; Zimarina et al., 2004; Doherty et al., 2005; Tao et al., 2006; Liu et al., 2007; Szyllo et al., 2007; Zhao et al., 2007; Hirata et al., 2008a; Li et al., 2010), which were composed of five papers in English (McGrath et al., 2004; Zimarina et al., 2004; Doherty et al., 2005; Tao et al., 2006; Hirata et al., 2008a), three in Chinese (Liu et al., 2007; Zhao et al., 2007; Li et al., 2010), and one in Polish (Szyllo et al., 2007) independently translated by a research assistant.

The main characteristics of these studies are listed in Table 2. In the nine studies, four studies involved Caucasians (McGrath et al., 2004; Zimarina et al., 2004; Szyllo et al., 2007; Hirata et al., 2008a), four involved Asians (Tao et al., 2006; Liu et al., 2007; Zhao et al., 2007; Li et al., 2010), and the other one involved Caucasian and African populations (Doherty et al., 2005). As for sample sources, three papers were populationbased (Doherty et al., 2005; Tao et al., 2006; Hirata et al., 2008a) and six were hospitalbased (McGrath et al., 2004; Zimarina et al., 2004; Liu et al., 2007; Szyllo et al., 2007; Zhao et al., 2007; Li et al., 2010). The sample sizes ranged from 164 to 2057 subjects. The genotype distributions of the COMT Val158Met in endometrial cancer cases and controls are shown in Table 3. Moreover, two studies provided detailed data of the premenopausal and postmenopausal subpopulations (McGrath et al., 2004; Tao et al., 2006), and one study discussed postmenopausal detection (Szyllo et al., 2007). The genotype frequencies of the controls from eight studies were in agreement with Hardy-Weinberg equilibrium (HWE), the one study (Liu et al., 2007) deviating from HWE was excluded in the final metaanalysis as indicated in Figure 1. Then, according to the predefined quality score rules in 
Table 1, five studies (McGrath et al., 2004; Zimarina et al., 2004; Doherty et al., 2005; Tao et al., 2006; Li et al., 2010) with $>200$ subjects, using blood for genotyping, and with a quality score of $>10$ were classified as high quality, whereas the other three (Szyllo et al., 2007; Zhao et al., 2007; Hirata et al., 2008a) with relative small sample size, using tissue DNA for genotyping, and deviating from HWE in controls with a score of $<10$ were labeled as low quality. Ultimately, eight eligible studies with 2296 endometrial cancer cases and 2813 controls were collected to perform meta-analysis in this study.

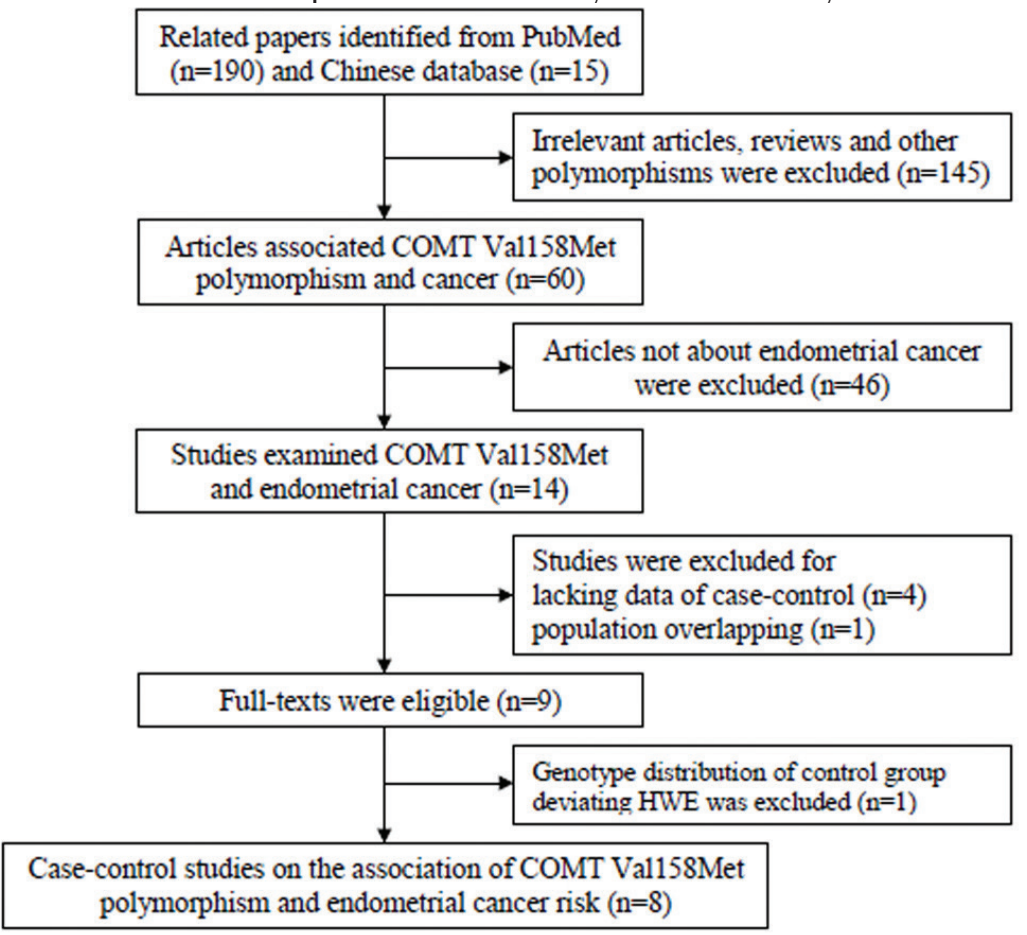

Figure 1. Flow chart of study selection explaining the eight eligible case-control studies included in the metaanalysis.

Table 2. Main characteristics of studies included in this meta-analysis.

\begin{tabular}{|c|c|c|c|c|c|c|c|c|}
\hline $\begin{array}{l}\text { First author } \\
\text { reference }\end{array}$ & Year & Country & Ethnicity & $\begin{array}{l}\text { Sample source } \\
\text { (cases/controls) }\end{array}$ & $\begin{array}{l}\text { Specimens } \\
\text { of cases }\end{array}$ & $\begin{array}{c}\text { Sample size } \\
\text { (cases/controls) }\end{array}$ & $\begin{array}{l}\text { Genotyping } \\
\text { method }\end{array}$ & $\begin{array}{l}\text { Quality } \\
\text { score }\end{array}$ \\
\hline Hirata & $2008 \mathrm{a}$ & USA & Caucasians & Hospital/population & Tissue & $150 / 165$ & $\begin{array}{l}\text { PCR-RFLP and } \\
\text { DNA sequencing }\end{array}$ & $<10$ \\
\hline Szyllo & 2007 & Polish & Caucasians & Hospital/hospital & Tissue & $151 / 197$ & PCR-RFLP & $<10$ \\
\hline Zhao & 2007 & China & Asian & Hospital/hospital & Tissue and blood & $132 / 110$ & PCR-RFLP & $<10$ \\
\hline Tao & 2006 & China & Asian & Population/population & $\begin{array}{l}\text { Blood and exfoliated } \\
\text { buccal cells }\end{array}$ & d $1031 / 1026$ & Taqman & $>10$ \\
\hline Doherty & 2005 & USA & $\begin{array}{l}\text { Caucasian and } \\
\text { African }\end{array}$ & Population/population & Blood & $371 / 420$ & PCR-RFLP & $>10$ \\
\hline Zimarina & 2004 & $\begin{array}{l}\text { Russia and } \\
\text { Norway }\end{array}$ & Caucasians & Hospital/hospital & Blood & $124 / 140$ & PCR-RFLP & $>10$ \\
\hline McGrath & 2004 & USA & Caucasians & Hospital/hospital & Blood & $215 / 641$ & PCR-RFLP & $>10$ \\
\hline Liu & 2007 & China & Asian & Hospital/hospital & Tissue & $80 / 84$ & PCR-RFLP & $<10$ \\
\hline $\mathrm{Li}$ & 2010 & China & Asian & Hospital/hospital & Blood & $122 / 114$ & PCR-RFLP & $>10$ \\
\hline
\end{tabular}




\begin{tabular}{|c|c|c|c|c|c|c|c|}
\hline \multirow[t]{3}{*}{ First author reference } & \multicolumn{7}{|c|}{ Genotype } \\
\hline & \multicolumn{3}{|c|}{ Cases } & \multicolumn{3}{|c|}{ Controls } & \multirow{2}{*}{$\frac{\text { HWE }}{\text { Controls }}$} \\
\hline & $\mathrm{Val} / \mathrm{Val}(\mathrm{N} \%)$ & Val/Met (N\%) & Met/Met (N\%) & Val/Val (N\%) & Val/Met (N\%) & Met/Met (N\%) & \\
\hline Hirata & $32(21.3)$ & $81(54.0)$ & $37(24.7)$ & $48(29.1)$ & $90(54.5)$ & $27(16.4)$ & 0.16 \\
\hline Szyllo & $46(30.5)$ & $81(53.6)$ & 24 (15.9) & $48(24.4)$ & $110(55.8)$ & $39(19.8)$ & 0.09 \\
\hline Zhao & $39(29.5)$ & $77(58.3)$ & $16(12.1)$ & $52(47.3)$ & $50(45.5)$ & $8(7.3)$ & 0.39 \\
\hline Tao & $563(54.6)$ & $383(37.1)$ & $85(8.2)$ & $534(52.0)$ & $425(41.4)$ & $67(6.5)$ & 0.15 \\
\hline Doherty & $97(26.1)$ & $174(46.9)$ & $100(27.0)$ & $90(21.4)$ & $207(49.3)$ & $123(29.3)$ & 0.87 \\
\hline Zimarina & $29(23.4)$ & $65(52.4)$ & $30(24.2)$ & $23(16.4)$ & $73(52.1)$ & $44(31.4)$ & 0.43 \\
\hline McGrath & $55(25.6)$ & $105(48.8)$ & $55(25.6)$ & $161(25.1)$ & $308(48.0)$ & $172(26.8)$ & 0.33 \\
\hline LIU & $42(52.5)$ & $33(41.3)$ & $5(6.3)$ & $35(41.7)$ & $46(54.8)$ & $3(3.6)$ & $0.01 *$ \\
\hline LI & $90(78.3)$ & $26(21.3)$ & $6(4.9)$ & $71(62.3)$ & $35(30.7)$ & $8(7.0)$ & 0.22 \\
\hline \multicolumn{8}{|l|}{ Premenopausal } \\
\hline Tao & $229(51.2)$ & $176(39.4)$ & $42(9.4)$ & $204(51.8)$ & $160(40.6)$ & $30(7.6)$ & 0.86 \\
\hline McGrath & $11(20.4)$ & $29(53.7)$ & $14(25.9)$ & $23(24.5)$ & $48(51.1)$ & $23(24.5)$ & 0.84 \\
\hline \multicolumn{8}{|l|}{ Postmenopausal } \\
\hline Tao & $334(57.2)$ & 207 (35.4) & $43(7.4)$ & $330(52.2)$ & 265 (41.9) & $37(5.9)$ & 0.09 \\
\hline McGrath & $43(27.4)$ & 73 (46.5) & $41(26.1)$ & $134(25.1)$ & $253(47.5)$ & $146(27.4)$ & 0.25 \\
\hline Szyllo & $46(30.5)$ & $81(53.6)$ & $24(15.9)$ & $48(24.4)$ & $110(55.8)$ & $39(19.8)$ & 0.09 \\
\hline
\end{tabular}

Val $=$ Valine; Met $=$ Methionine $; *$ Deviation from HWE.

\section{Meta-analysis results}

The main results of this meta-analysis are listed in Table 4. No significant associations between COMT Val158Met polymorphism and endometrial cancer susceptibility were observed when the studies were pooled, in all the genetic models. However, in stratified analysis based on menopausal status and study quality, reduced risk of endometrial cancer were found in case of Val/Met versus Val/ Val and Met/Met + Val/Met versus Val/Val comparisons for the postmenopausal subpopulation (Val/ Met versus Val/Val: $\mathrm{OR}=0.795,95 \% \mathrm{CI}=0.656-0.962, \mathrm{P}=0.019 ; \mathrm{Met} / \mathrm{Met}+\mathrm{Val} / \mathrm{Met}$ versus Val $/$ Val: $\mathrm{OR}=0.819,95 \% \mathrm{CI}=0.683-0.983, \mathrm{P}=0.032$; as shown in Figure $2 \mathrm{~A}$ and $\mathrm{B}$ ), and similar results also existed in the five high-quality studies (Val/Met versus Val/Val: $\mathrm{OR}=0.835,95 \% \mathrm{CI}=0.726$ $0.961, \mathrm{P}=0.012 ; \mathrm{Met} / \mathrm{Met}+\mathrm{Val} / \mathrm{Met}$ versus $\mathrm{Val} / \mathrm{Val}: \mathrm{OR}=0.853,95 \% \mathrm{CI}=0.747-0.974, \mathrm{P}=0.019$; as shown in Figure $2 \mathrm{C}$ and $\mathrm{D}$ ). When subgroup analysis based on ethnicity and source of controls was performed, no evidence of associations was discovered among these subpopulations.

\section{Sensitivity analyses}

Influence analysis was performed to determine the effect of individual data on the pooled OR by omitting one study at a time when repeating the meta-analysis. Most of the corresponding pooled OR were not significantly altered (data not shown).

\section{Publication bias}

Both Begg's funnel plot and Egger's tests were employed to detect publication bias of the literature collected. Begg's funnel plots did not find any obvious asymmetry in any meta-analysis (figures not shown). Then, Egger's test was performed to gather statistical evidence of funnel plot symmetry, which still did not show any obvious publication bias in all genetic models studied, such as Val/Met versus Val/Val: Begg's test $\mathrm{P}=0.902$ and Egger test $\mathrm{P}=0.616$; Met/Met $+\mathrm{Val} / \mathrm{Met}$ versus Val/Val: Begg's test $\mathrm{P}=0.902$ and Egger test $\mathrm{P}=0.798$; Val/Val + Met/Met versus Val/Met: Begg's test $\mathrm{P}=0.386$ and Egger test $\mathrm{P}=0.352$. These analyses indicate that our results are statistically robust. 
Association of COMT Val158Met and endometrial cancer risk

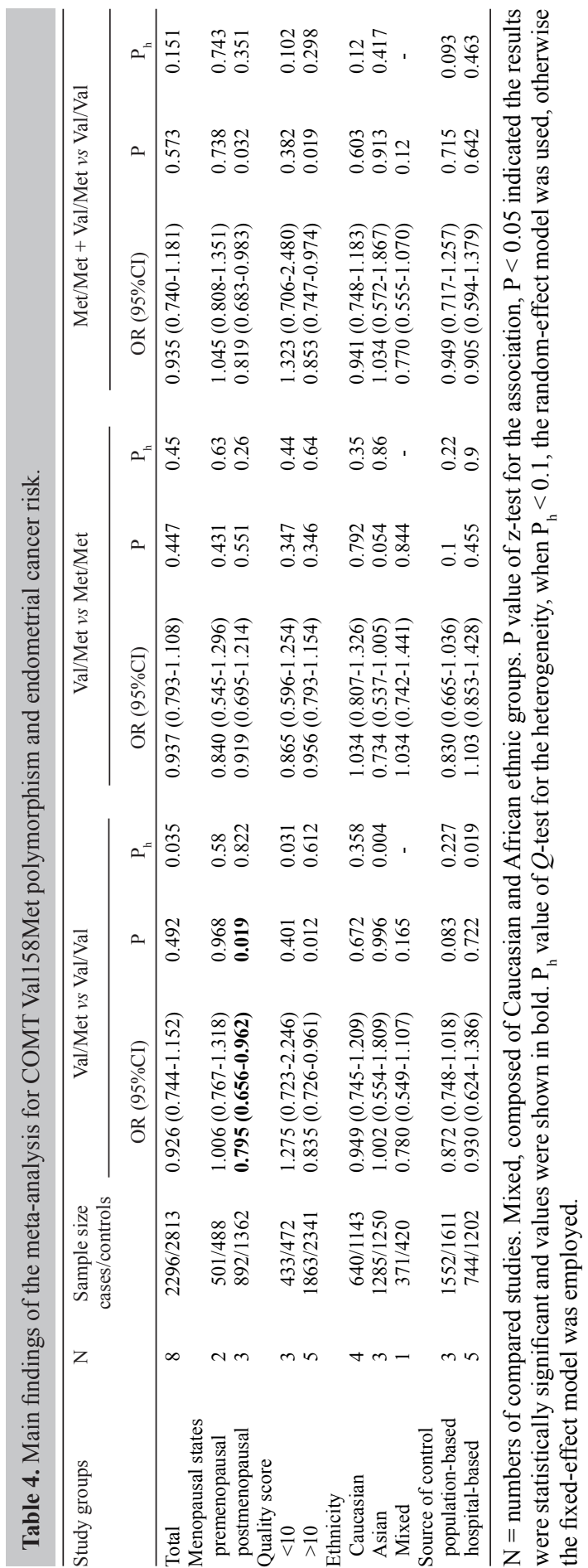




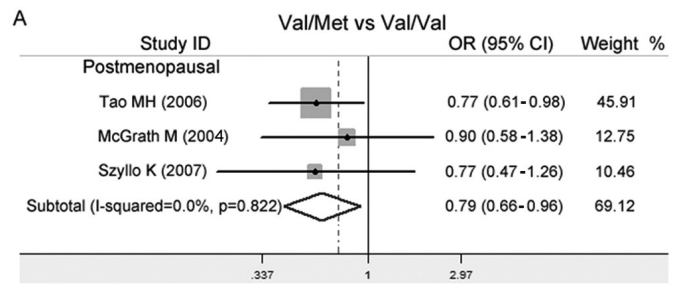

C

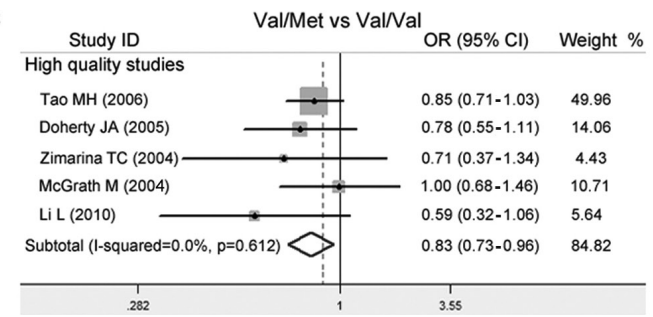

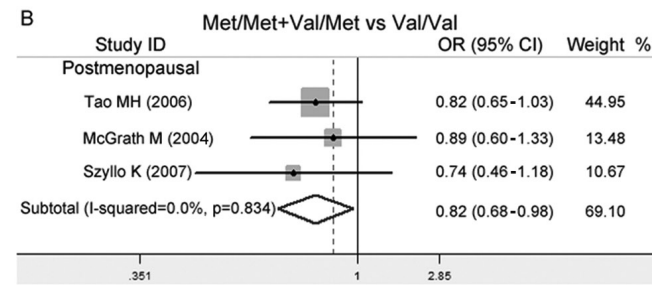

D

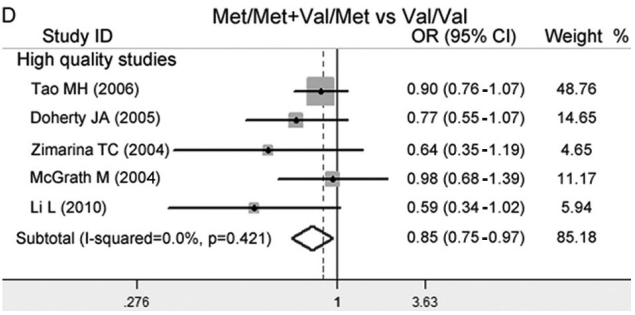

Figure 2. Forest plots of the association between COMT Val158Met polymorphism and endometrial cancer risk, for Val/Met versus Val/Val and Met/Met + Val/Met versus Val/Val genetic models via meta-analysis in the postmenopausal subgroup ( $\mathbf{A}$ and $\mathbf{B}$ ) and in the high-quality studies $(\mathbf{C}$ and $\mathbf{D})$, respectively. The gray square centers and corresponding horizontal lines represent the study-specific OR and the accompanying 95\% CI. The area of each square reflects its proportion to the sample size and the weight used in this analysis. All subtotal OR were calculated with fixed-effects models, and are presented by hollow diamonds.

\section{DISCUSSION}

Estrogens have long been thought to be involved in the pathogenesis of many diseases such as Alzheimer's syndrome, hypertension, breast cancer, endometrial cancer, etc. The following mechanisms may be involved. First, estradiol, the main member of estrogens, is a pleiotropic transcription factor involved in the activation of many disease-related genes with response elements (EREs) after binding to the estrogen receptor (ER) (Kushner et al., 2000). Second, the metabolites of estrogens have also closely been correlated with the development of various syndromes and cancers (Cavalieri et al., 1997). For instance, the accumulation of catechol estrogens may induce DNA damage and cause mutations in some pivotal genes. COMT is an important enzyme to inactivate carcinogenic catechol estrogens by methylation modification and to inhibit tumor initiation. COMT gene locates on the chromosome 22q11.2, contains six exons, and expresses in various mammalian tissues including the liver, kidney, endometrium, and breast, at high levels (Lundstrom et al., 1995). There are some singlenucleotide polymorphisms (SNPs) in the COMT gene, as summarized by the SNP500 Cancer Database (Packer et al., 2006): COMT SNPs in codon 72 of exon 3 (rs6267) and codon 102 of exon 4 (rs5031015) are very rare in Caucasians, whereas the SNPs in codon 62 (rs4633), 136 (rs4818), and 158 (rs4680) are common (http://snp500cancer.nci.nih.gov). As the polymorphism of the COMT codon 158 leads to Val-to-Met substitution, and the Met carriers may partially lose the methylation activity (Dawling et al., 2001), the COMT Val158Met has been extensively investigated for correlation with tumor risk, including in case of endometrial cancer. However, the results of recent studies are inconsistent for different races. One study involving women in South China found that the COMT Val/Val carriers may decrease the 
endometrial cancer risk compared with the COMT Met/Met genotype (Zhao et al., 2007); another study involving Caucasian and African American populations showed that low-activity COMT Val/Met and Met/Met carriers have a slightly decreased risk (Doherty et al., 2005). Other studies involving American (McGrath et al., 2004; Hirata et al., 2008a), Polish (Szyllo et al., 2007), Chinese (Tao et al., 2006; Liu et al., 2007; Li et al., 2010), Russian, and Norwegian populations (Zimarina et al., 2004) did not observe significantly different distribution of the COMT Val158Met genotype in the endometrial cancer patients and controls.

To resolve the above issues stemming from COMT Val158Met polymorphism and endometrial cancer risk, we summarized these studies and performed meta-analysis for 2296 cases and 2813 controls to evaluate the overall effects. Our results indicate that Val158Met is not correlated with endometrial cancer susceptibility in pooled analysis, which suggest that the COMT Val158Met polymorphism alone may be insufficient to predict the risk, and the effects of other COMT regulatory variations, COMT haplotypes, and CpG hypermethylation of the COMT promoter should be considered. Moreover, women developing endometrial cancer often have high estradiol levels that can inhibit COMT expression (Xie et al., 1999) and may outweigh the activity difference resulting from Val158Met polymorphism. However, stratified analysis according to menopausal status, an important risk factor for endometrial cancer pathogenesis, showed that $\mathrm{Val} / \mathrm{Val}$ plays obviously negative roles versus $\mathrm{Val} / \mathrm{Met}(\mathrm{P}=0.019)$ and Met/Met + Val/Met $(\mathrm{P}=0.032)$ in the postmenopausal women. This result is consistent with that of a previous study about COMT Val158Met on breast cancer risk (Thompson et al., 1998); they also found that COMT Val/Val genotype associated with increasing breast cancer risk in postmenopausal women. These data indicate the presence of complex interactions between COMT Val158Met and menopausal status in hormone-related cancers. In addition, the associations also existed among well-designed studies, Val/Val genotype may significantly increase endometrial cancer risk compared with Val/Met $(\mathrm{P}=0.012)$ and $\mathrm{Met} / \mathrm{Met}+\mathrm{Val} / \mathrm{Met}$ carriers $(\mathrm{P}=0.019)$. Our classification of high-quality studies is helpful to minimize bias, enhance statistical power, and obtain plausible conclusions. However, we did not find evidence of the association between Val158Met and endometrial cancer in Asians, Caucasians, and mixed populations, suggesting that the genetic backgrounds of people belonging to different ethnicities and environments have little impact on the correlation. Although COMT Val158Met polymorphism may not act as an independent factor for predicting cancer risk, the correlation can be detected in some specific subpopulations such as in postmenopausal women.

Meanwhile, there are some limitations of this meta-analysis. First, the control sources were not uniform. Most hospital-based controls had endometrial diseases and some of them might have been at risk to develop cancer in future, although our results from both populationand hospital-based controls showed no difference. Second, we performed only unadjusted evaluation on the association, because some detailed data such as BMI (body mass index), estrogen administration, and smoking status were not available, and therefore, these potential interactions could not be further estimated. Third, meta-analysis is the retrospective research, which is subject to some methodological deficiencies. To minimize the bias, we followed the previous quality score system (Jiang et al., 2010) and classified five high-quality and three low-quality studies. Although the predefined quality assessment have considered the traditional epidemiological and oncological issues, and the scoring was performed independently by two investigators to reduce the subjective effects, more standard scoring procedures for such studies are still expected to be established in future. 
In conclusion, our results suggest that COMT Val/Val carriers may significantly increase endometrial cancer risk in postmenopausal women, which may help us identify high-risk subpopulation and create more effective strategies for prevention and treatment. Further studies may be required to address the possible effects of different life styles and environmental factors on the association between COMT Val158Met polymorphism and endometrial cancer risk.

\section{ACKNOWLEDGMENTS}

Research supported by the Natural Science Foundation of China (\#81201521), the Natural Science Foundation of Shanghai for Young Scientist (\#12ZR1441500), the Key Technologies Research and Development Program for Infectious Diseases of China (\#2012ZX10003-006), and the Key Project of Science and Technology of Shanghai (\#10411955000).

\section{REFERENCES}

Ball P, Knuppen R, Haupt M and Breuer H (1972). Interactions between estrogens and catechol amines. 3. Studies on the methylation of catechol estrogens, catechol amines and other catechols by the ctechol-O-methyltransferases of human liver. J. Clin. Endocrinol. Metab. 34: 736-746.

Begg CB and Mazumdar M (1994). Operating characteristics of a rank correlation test for publication bias. Biometrics 50: 1088-1101.

Cavalieri EL, Stack DE, Devanesan PD, Todorovic R, et al. (1997). Molecular origin of cancer: catechol estrogen-3,4quinones as endogenous tumor initiators. Proc. Natl. Acad. Sci. U. S. A. 94: 10937-10942.

Dawling S, Roodi N, Mernaugh RL, Wang X, et al. (2001). Catechol-O-methyltransferase (COMT)-mediated metabolism of catechol estrogens: comparison of wild-type and variant COMT isoforms. Canc. Res. 61: 6716-6722.

DerSimonian R and Laird N (1986). Meta-analysis in clinical trials. Control Clin. Trials 7: 177-188.

Doherty JA, Weiss NS, Freeman RJ, Dightman DA, et al. (2005). Genetic factors in catechol estrogen metabolism in relation to the risk of endometrial cancer. Canc. Epidemiol. Biomarkers Prev. 14: 357-366.

Egger M, Davey SG, Schneider M and Minder C (1997). Bias in meta-analysis detected by a simple, graphical test. BMJ 315: 629-634.

He XF, Wei W, Li SX, Su J, et al. (2012). Association between the COMT Val158Met polymorphism and breast cancer risk: a meta-analysis of 30,199 cases and 38,922 controls. Mol. Biol. Rep. 39: 6811-6823.

Higgins JP, Thompson SG, Deeks JJ and Altman DG (2003). Measuring inconsistency in meta-analyses. BMJ 327: 557-560.

Hirata H, Hinoda Y, Okayama N, Suehiro Y, et al. (2008a). COMT polymorphisms affecting protein expression are risk factors for endometrial cancer. Mol. Carcinog. 47: 768-774.

Hirata H, Hinoda Y, Okayama N, Suehiro Y, et al. (2008b). CYP1A1, SULT1A1, and SULT1E1 polymorphisms are risk factors for endometrial cancer susceptibility. Cancer 112: 1964-1973.

Huber JC, Schneeberger C and Tempfer CB (2002). Genetic modelling of the estrogen metabolism as a risk factor of hormone-dependent disorders. Maturitas 41 (Suppl 1): S55-S64.

Jiang DK, Ren WH, Yao L, Wang WZ, et al. (2010). Meta-analysis of association between TP53 Arg72Pro polymorphism and bladder cancer risk. Urology 76: 765-767.

Kushner PJ, Agard DA, Greene GL, Scanlan TS, et al. (2000). Estrogen receptor pathways to AP-1. J. Steroid Biochem. Mol. Biol. 74: 311-317.

Li L, Li FX, Zhang N and Du YF (2010). Association of gene polymorphism in cytochrome P450 1 B1 and COMT, with the expression of mRNA and susceptibility to endometrial cancer in Chinese. J. Hebei Med. Univ. 31: 1433-1437.

Liu J, Yang XS, Qu X and Li H (2007). Relation among single nucleotide metablolizing genes CYP17, COMT and endometrial adenocarcinoma risk in Chinese. J. Shandong Univ. 45: 18-21.

Lundstrom K, Tenhunen J, Tilgmann C, Karhunen T, et al. (1995). Cloning, expression and structure of catechol-Omethyltransferase. Biochim. Biophys. Acta 1251: 1-10.

Mantel N and Haenszel W (1959). Statistical aspects of the analysis of data from retrospective studies of disease. J. Natl. Canc. Inst. 22: 719-748.

McGrath M, Hankinson SE, Arbeitman L, Colditz GA, et al. (2004). Cytochrome P450 1B1 and catechol-O- 
methyltransferase polymorphisms and endometrial cancer susceptibility. Carcinogenesis 25: 559-565.

Packer BR, Yeager M, Burdett L, Welch R, et al. (2006). SNP500Cancer: a public resource for sequence validation, assay development, and frequency analysis for genetic variation in candidate genes. Nucleic Acids Res. 34: D617-D621.

Sorosky JI (2008). Endometrial cancer. Obstet. Gynecol. 111: 436-447.

Suzuki M, Kurosaki T, Arai T, Sawabe M, et al. (2007). The Val158Met polymorphism of the catechol-O-methyltransferase gene is not associated with the risk of sporadic or latent prostate cancer in Japanese men. Int. J. Urol. 14: 800-804.

Szyllo K, Smolarz B, Romanowicz-Makowska H, Przybylowska K, et al. (2007). The risk of endometrial cancer appearance and CYP19 and COMT gene polymorphism. Pol. Merkur. Lekarski 22: 208-210.

Tao MH, Cai Q, Xu WH, Kataoka N, et al. (2006). Cytochrome P450 1B1 and catechol-O-methyltransferase genetic polymorphisms and endometrial cancer risk in Chinese women. Canc. Epidemiol. Biomarkers Prev. 15: 2570-2573.

Thompson PA, Shields PG, Freudenheim JL, Stone A, et al. (1998). Genetic polymorphisms in catechol-Omethyltransferase, menopausal status, and breast cancer risk. Canc. Res. 58: 2107-2110.

Woolf B (1955).On estimating the relation between blood group and disease. Ann. Hum. Genet. 19:251-253.

Xie T, Ho SL and Ramsden D (1999). Characterization and implications of estrogenic down-regulation of human catecholO-methyltransferase gene transcription. Mol. Pharmacol. 56: 31-38.

Zhao XM, Xie MQ, Yang DZ, Wang LA, et al. (2007). Polymorphism of catechol-O-methyltransferase gene in relation to the risk of endometrial cancer. Zhonghua Fu Chan Ke Za Zhi. 42: 116-119.

Zhu BT and Conney AH (1998a). Functional role of estrogen metabolism in target cells: review and perspectives. Carcinogenesis 19: 1-27.

Zhu BT and Conney AH (1998b). Is 2-methoxyestradiol an endogenous estrogen metabolite that inhibits mammary carcinogenesis? Canc. Res. 58: 2269-2277.

Zimarina TC, Kristensen VN, Imianitov EN and Bershtein LM (2004). Polymorphisms of CYP1B1 and COMT in breast and endometrial cancer. Mol. Biol. 38: 386-393. 